\title{
Models of Quasi-Steady and Unsteady Discharge from Plumbing Fixtures
}

\author{
Bal M. Mahajan, * Lawrence S. Galowin, * and Paul A. Kopetka* \\ Notional Bureau of Standards, Washington, DC 20234
}

August 20, 1980

\begin{abstract}
Modeling methods are developed to predict the discharge characteristics of simulated simplified configurations for plumbing fixtures connected to horizontal drain branch piping. Computations are carried out to illustrate several methods of determining the effect of various loss coefficients of the drain connection, pipe length, pipe diameter, and friction factor. Solutions are obtained for the case of a fixture with a constant head (continuous refill) and a falling head (emptying of a sink). Numerical solution of the non-linear differential equation for the falling head case was obtained by the Runge-Kutta method. Discharge characteristics are presented for a range of flows and pipe diameter-to-length ratios representative of plumbing installations. The feasibility of developing predictive models for hydraulic characteristics of interconnected plumbing fixture and drainage piping systems is shown. The variations of efflux rate with the drain pipe diameter, length, and slope obtained from the assumed models are similar in trend to the available experimental data.
\end{abstract}

Key words: characteristics; discharge; drainage; flow; model; plumbing; quasi-steady; unsteady.

\section{Nomenclature}

$A=$ cross-sectional area of the container

$a=$ cross-sectional area of the pipe

$d=$ pipe diameter

$f=$ pipe friction factor

$g=$ acceleration due to gravity

$h=$ instantaneous elevation of the free surface in the container above the center of the outlet of the drain

$h_{o}=$ initial value of $h$

$h_{e}=$ initial elevation of the free surface in the container above the center of the outlet section of initial pipe or inlet section of drain pipe (see fig. 3)

$k_{l}=$ loss coefficient for the tank drain connection

$k_{b}=$ loss coefficient for the initial pipe bends and the initial pipe-exit pipe coupling

$R_{c}=$ loss coefficient for the sudden change in pipe diameter at the location where the initial pipe is coupled to the drain pipe

$Q_{i}=a_{i} \mathrm{U}_{i}=$ ideal efflux rate, i.e., the efflux rate when all head losses are neglected

$Q_{e}=$ efflux rate $=a_{e} U_{e}$

$u=$ instantaneous flow velocity

$U_{e}=$ mean velocity of flow in the exit pipe

$U_{i}=$ mean ideal velocity of flow in the exit pipe, that is the velocity when all head losses are neglected

$p=$ pressure
$P_{a}=$ atmospheric pressure

$s=\tan \alpha=$ slope or pitch of the drain pipe

$\alpha=$ drain pipe slope angle

$\beta=d_{e} / d_{i}$

$\gamma=$ specific weight of water

$\phi=\left(1+k_{1}+f L / d\right)$

$\phi_{1}=\left(k_{1}+K_{b}+f_{1} L_{l} / d i\right)$

$\phi_{2}=\left(f_{e} L_{e} / d_{e}+k_{c}\right)$

Subscript

1 refers to initial pipe

$e$ refers to drain pipe

Metric Conversion

feet $\times 0.3048=$ meters

\section{Introduction}

The mathematical modeling for determining the time dependent capacity characteristics of the drain-waste-vent plumbing system requires that the prescribed wastewater loads from a variety of fixtures and devices be determinable as the initial and boundary conditions. The development of models for calculation of time dependent discharge flows from plumbed fixtures as the forcing functions required to describe the loads on the drainage system is not well estab-

- Center for Building Technology, National Engineering Laboratory. 
lished. The dynamics of the fixture discharge loading are mostly derived from experimental data sources that provide the combined loss effects as empirical factors. The theoretical developments are rendered difficult by the complex geometric configuration of the connecting elements of the fixture-drainage system (e.g. traps, elbows, tees, overflow drain, stopper flow area control, and branch drains) and by the turbulent flow conditions and secondary swirling. Further difficulties in a complete theoretical development are encountered due to the transition from full pipe flow to partially filled pipe flow downstream of the trap in the horizontal branch where changes in the size of elbow fittings, pipe diameter and slope often occur.

The dynamic transient characteristics of connected fixtures are generally assumed to be, a priori, independent of the downstream connections, drain or stopper geometry, elbow, fittings and the branch piping [1, 2]. ${ }^{1}$ Pressure variations within the drainage system (propagation of downstream conditions typical of incompressible low flow phenomena) are neglected in the usual methods for testing fixture performance in open air discharge tests at atmospheric pressure. The pressure variations, above and below atmospheric, occur in multi-story buildings as shown by Konen [3] and can influence fixture outflow.

Requirements for sizing the drain system as embodied in the plumbing codes in the U.S. are based upon the concepts of "fixture units." This traditional approach for water supply pipe sizing was extended to drainage systems, since the outflow very nearly equals inflow. This method, however, does not introduce the transient dynamics of the discharge flow phenomena and is applied as a "quasi-steady-state" loading for pipe sizing; it does, however, provide the significant input for peak water loads.

Test data comparing isolated open air fixture discharge characteristics with pipe connected fixtures show that the discharge curves are influenced by the connections to the drainage system; the predictive models should include the relevant constraints due to these connections. The model adopted in this study is for a horizontal drain carrying waste water from a fixture. The fixture discharge characteristics are found to be modified when losses due to piping and connections are introduced in the flow equations. The characteristics of interest are peak efflux rate, efflux ratetime history, maximum flow velocity in the horizontal drain, velocity-time history and the time required to empty the fixture. The parameters of interest for fixtures are the volume of water in the fixture, geometrical shape of the fixture, fixture to drain connections, drain exit conditions and physical characteristics of the drain pipe (i.e. diameter, length, slope, and friction factor).

\footnotetext{
- Figures in brackets indicate literature references at the end of this paper.
}

This investigation is intended to be a parametric study only, varying each characteristic, one at a time; subsequent experiments are required to provide specific loss factors.

The plumbing fixtures are connected to the horizontal drains via connecting elements such as traps, downpipes, elbows, tees and other pipe fittings. The fixture discharge characteristics are influenced by the pressure losses and energy dissipation in these connecting elements, and by the physical characteristics of drain pipe. Predictive mathematical models and experimental validations are required to determine the effects of connecting elements and drain pipe characteristics on the fixture discharge parameters. The discharge characteristics of various plumbing fixtures with drain pipes of different physical characteristics (i.e. diameter, length and slope) was reported by Hanslin and Perrier [4] in an experimental investigation. Classical approaches based upon fundamentals and overly simplified models concerning the effects of the variables on the efflux rate from fixtures are available in basic hydraulic texts [ 5 and 6]. However, these studies for predicting the discharge characteristics of plumbing fixtures did not utilize representative models for effects of connector variables on the discharge characteristics.

This report illustrates the development of mathematical models for predicting the transient and quasi-steady state hydraulic discharge characteristics of a fixture coupled to the piping of a plumbing systems. This effort is the first step in developing the details of the dissipation effects from losses experienced in the flow within plumbing fixtures and connecting pipes. The next significant effort is required to develop a detailed analytical basis for the partially filled horizontal drain pipe. The effects of drain pipe parameters on the discharge characteristics of an open container, such as a lavatory or sink, under different flow conditions are presented. The number of variables for each model studied have been reduced in order to illustrate the most significant effects. For the steady state efflux rate from a constant head (continuous refill) container the effects of drain pipe parameters, taken one at a time, are shown. Also the solution for emptying a fixture based upon the instantaneous efflux rate from a falling head container (emptying fixture) is presented.

\section{Unsteady flow drain-Falling head}

Consider an open container with the uniform diameter drain pipe of length $L$ fitted with a quick opening valve as shown in figure 1 to simulate a lavatory branch drainage pipe. Initially the container is full and the valve is closed. When the valve is rapidly opened, the available head will accelerate the fluid, the velocity of flow and rate of efflux will increase from zero to a maximum value. After attaining the maximum value, the flow velocity will decrease, because 


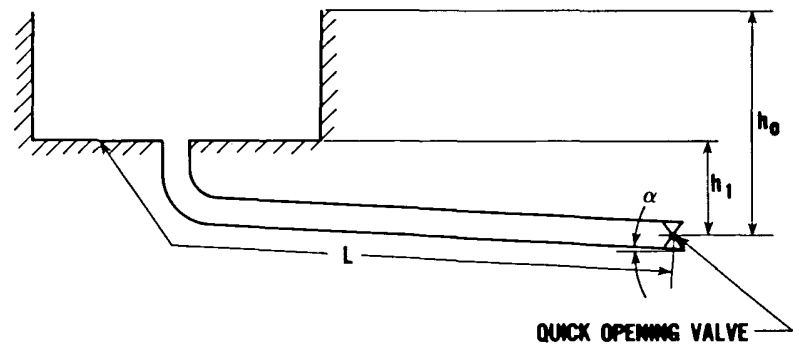

FIGURE 1. Lavatory-branch drain schematic.

the elevation of the water surface in the container will be decreasing as the water leaves the container. The final outflow from the tank drain into the pipe would experience a discontinuity in the water surface area.

A one-dimensional unsteady flow analysis is adopted that leads to the non-linear equation of motion of the liquid discharged from a simulated lavatory-branch drainage pipe. The one-dimensional method assumes a uniform velocity across the flow area. Resistance and dissipation such as shear at the wall, turbulent mixing and drain vortex formation, and shedding losses are introduced through an empirical quasi-steady state set of constants. The losses are assumed to be proportional to the square of the velocity.

The equation of motion for the one-dimensional flow is:

$$
\frac{L}{g} \frac{d u}{d t}=h(t)-\phi u^{2} / 2 g
$$

where: $h=$ instantaneous elevation of the free surface above the center of the outlet section of the drain

$u=$ instantaneous flow velocity in the drain pipe

$\phi=$ dissipation factor

The forcing term on the right hand side of eq (1) causing acceleration or deceleration of the fluid is dependent upon the decreasing head and the dissipation function. In this simplified model the losses accounted for are the friction in the pipe, and the loss coefficient for the tank to drain connection $[5,7]$. The function is given by:

$$
\phi=1+k_{1}+f L / d
$$

where: $\quad k_{1}=$ loss coefficient for the tank drain connection

$f=$ friction factor for the pipe taken as a constant factor for the range of Reynolds numbers

$L / d=$ length to diameter ratio

The first term of eq (2) simply accounts for the decrease in total pressure head as a driving force due to the frictionless conversion to the dynamic pressure. The ideal frictionless fluid case results from the assumption of $k_{1}=f=0$.

The relationship between the velocity $u$, at the drain of cross-sectional area, a, and the head, $h(t)$, in the tank of cross-sectional area, $A$, is obtained from the continuity equation,

$$
\frac{d h}{d t}=-\frac{a}{A} u
$$

The minus sign accounts for the falling head in the direction of the velocity.

The simultaneous eqs (1) and (3) are combined by assuming $\phi$ independent of time, differentiating (1), substituting (3) for $d h / d t$ and rearranging the terms to yield

$$
d^{2} u / d t^{2}+\frac{\phi}{L} u \frac{d u}{d t}+\frac{a}{A} \frac{g}{L} u=0
$$

when

$$
h<h_{1}, a / A=1 \text {. }
$$

The initial conditions are:

$$
t=o, h=h_{o}, u=o, \frac{d u}{d t}=g h o / L
$$

with the last condition from eq (1). Equation (4) is recognized as a non-linear equation with constant coefficients when $\phi$ is assumed to take on quasi-steady values. The dissipation factors are functions of the local time dependent flow conditions; the terms of the function are taken as constant values over the flow regime, i.e. quasi-steady conditions, in this report. The solution by a numerical method based upon finite difference techniques is readily obtained.

The solution of eq (4) provides the flow velocity as a function of time. The numerical integration of the equation was obtained from a computer programmed Runge-Kutta method. A numerical integration method provides for adjustments in the value of $a / A, k$, and $f$ at various points in the stepwise calculation, if it is desired to account for their variations. For a container with constant cross-sectional area, $a / A$ changes only when the container empties and fluid is still present in the vertical section of the drain, i.e. when $\mathrm{h}$ is less than $h_{1}$.

Example discharge velocity variations with time are shown in figure 2. In each of the three samples shown the acceleration from rest is very nearly constant over the initial time to attain the maximum flow. Since the velocity, $u$, is shown to be linear, it can be inferred from eq (3) that the height, $h$, varies with square of the time, $t$. The predicted discharge characteristics resemble the experimental discharge characteristics for various plumbing fixtures published in the literature [ 8 and 9]. To obtain quantitative 


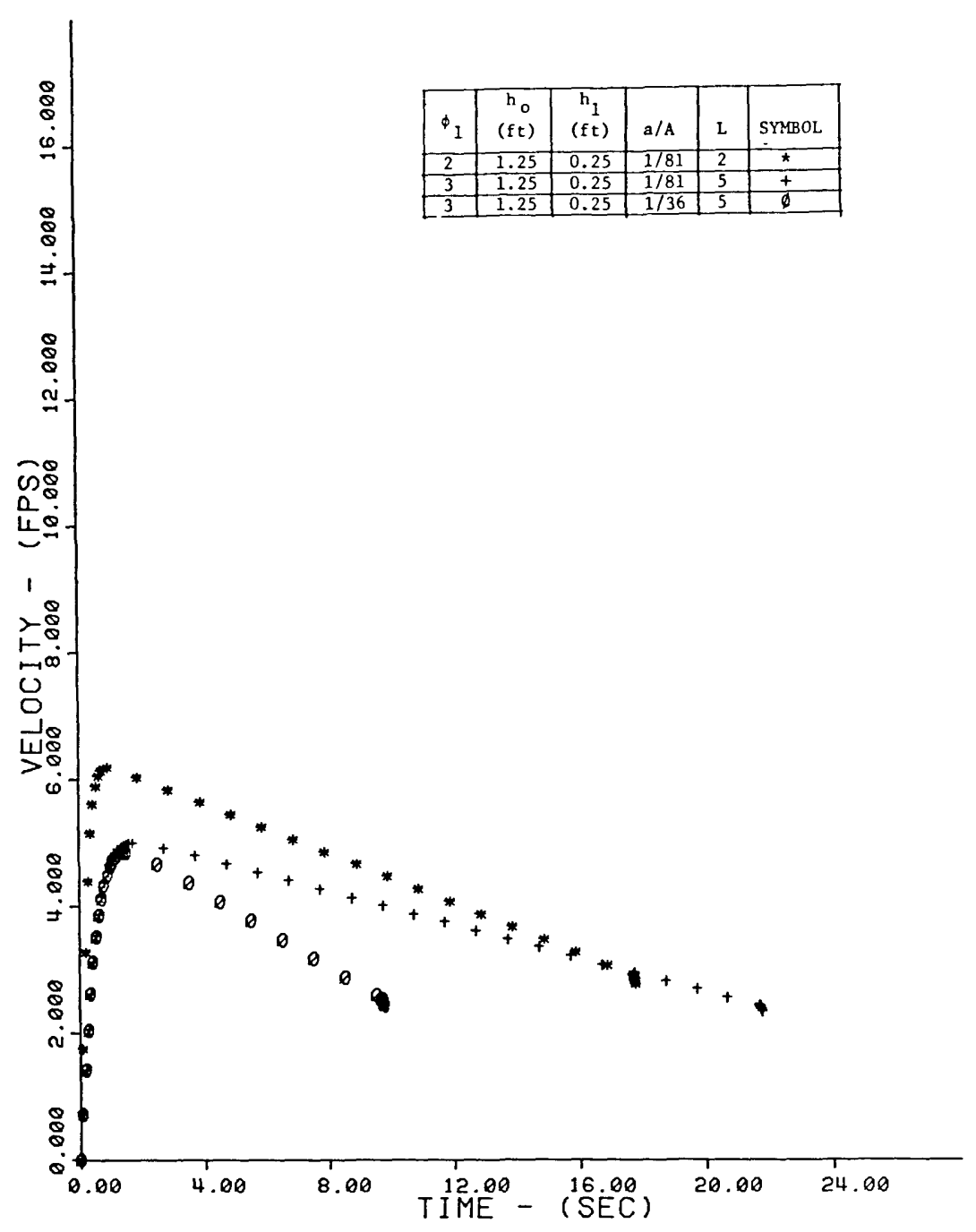

FigurE 2. Discharge characteristics of a container-drainage system.

agreement between the predicted and experimental results it is necessary to determine the values of various loss coefficients from the experiments. As anticipated, the smallest time of discharge is obtained for the larger drain area ratio when holding the other terms constant; the maximum efflux velocity occurs with the lowest dissipation values.

\section{Effects of drainage piping}

The dependence of the discharge characteristics upon the interconnections of fixtures and devices with the piping system and the fittings at drain connections are investigated. The schematic for the steady state efflux from a con- stant head (continuous refill) open container and drainage piping system is shown in figure 3 . The assumption of constant head refill condition simplified the problem to a steady state analysis. The governing equations do not require the development of computer based numerical solutions of the differential equations. The effects of the variables of the piping system and drain connections can then be more readily evaluated in a parametric manner.

The drainage system consists of an initial pipe of constant inside diameter $d_{1}$ and length $L_{1}$, the initial pipe is coupled to a drain pipe of constant diameter $d_{e}$, length $L_{e}$ and slope angle $\alpha$. The mean velocity of the flow $\left(U_{e}\right)$ in the drain pipe may be obtained by applying the modified Bernoulli equation between " $O$ " and " $e$ " as: 

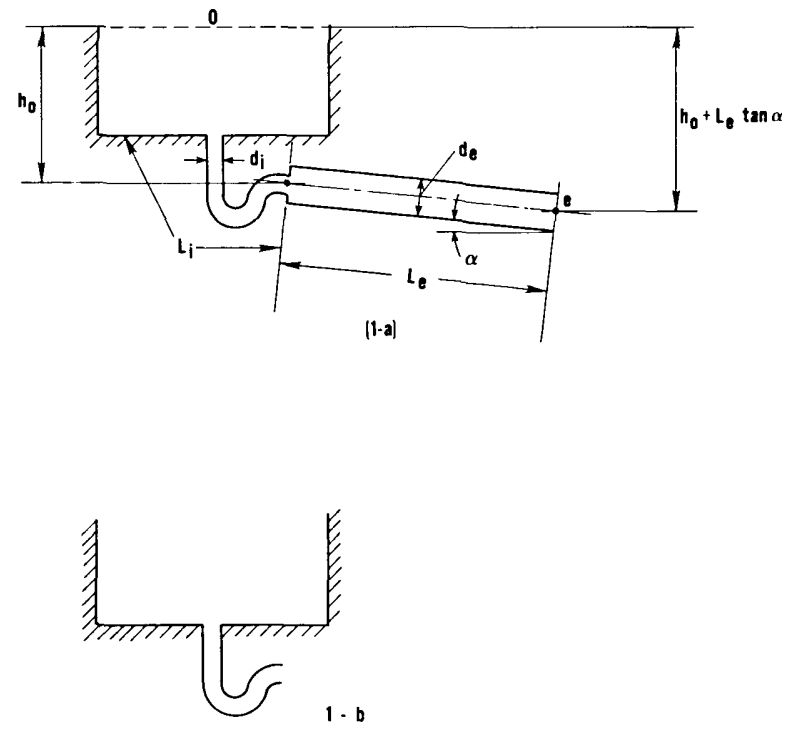

FIGURE 3. Constant head container drainage system.

$$
\begin{gathered}
P_{\circ} / \gamma+Z_{o} U_{o}^{2} / 2 g= \\
P e / \gamma+Z_{e}+U_{e}^{2} / 2 g+\phi_{1} U_{1}^{2} / 2 g+\phi_{2} U_{e}^{2} / 2 g
\end{gathered}
$$

with the conditions at inlet and outlet given by:

$$
P_{o}=P_{e}=P_{a} ; U_{o}=0 ; Z_{o}-Z_{e}=h_{e}-L_{e} s
$$

where $s=\sin \alpha$ and $U_{1}$ the velocity at the exit of the trap, i.e. the inlet of the drain pipe.

The dissipation functions are $\phi_{1}$ and $\phi_{2}$ where $\phi_{1}$ is the function representing the losses at the tank to drain connection and in the initial pipe, and $\phi_{2}$ is the function representing the losses at the step change in diameters and the horizontal drain. The equations are:

$$
\begin{aligned}
& \phi_{1}=\left(k_{1}+k_{b}+f_{1} L_{1} / d_{1}\right) \\
& \phi_{2}=\left(f_{e} / L_{e} / d_{e}+k_{c}\right)
\end{aligned}
$$

The continuity equation requires that for full bore pipe flow

$$
U_{1}=\beta^{2} U_{e}, \quad \beta^{2}=a_{e} / a_{1}
$$

and the flow rate

$$
Q_{e}=a_{e} U_{e}
$$

The condition of partially filled pipe flows is not considered here. A more extensive analysis is required to establish the transition condition for break away from full flow to partial filled flow.
The simplification of terms result in the velocity and flow rate equations

$$
U_{e}=U_{i}\left[\left(1+\frac{L_{e} s}{h_{0}}\right) /\left(1+\beta^{4} \phi_{1}+\phi_{2}\right)\right]^{1 / 2}
$$

or

$$
Q_{e}=Q_{i} \beta^{2}\left[\left(1+\frac{L_{e} s}{h_{o}}\right) /\left(1+\beta^{4} \phi_{1}+\phi_{2}\right)\right]^{1 / 2}
$$

where the symbols are defined in the nomenclature.

Let $U_{r}$ represent the mean velocity of flow and $Q_{r}$ the efflux rate when the drainage system of the container of figure 3-a consists of just the initial pipe as shown in figure $3 \mathrm{~b}$. The expressions for $U_{r}$ and $Q_{r}$, are obtained by letting $L_{e}$ and $\phi_{2}$ equal to zero and $\beta=1$ as:

$$
U_{r}=U_{i} /\left(1+\phi_{1}\right)^{1 / 2}
$$

and

$$
Q_{r}=U_{r} a_{1}=Q_{i} /\left(1+\phi_{1}\right)^{1 / 2} \text {. }
$$

Equations (5) through (9) show the dependence of $U_{e}$ and $Q_{e}$ on the drain pipe variables (i.e., $d_{e}, L_{e} s$, and $f_{e}$ ). For a falling head container where the conditions are slowly varying (i.e. area ratio of pipe to container less than 0.1 ), and may be considered as quasi-steady, these equations also give the instantaneous flow velocity and instantaneous efflux rate. The instantaneous flow velocity and efflux rate may be found by replacing $h_{o}$ with the instantaneous value of the height, $h$, of the free surface in the container above the exit center of the cross-section of the initial pipe.

Using eqs (8a), (9a), (8b) and (9b), the quantities $U_{\text {c and }} Q$. may be nondimensionalized as:

$$
\begin{gathered}
U_{e} / U_{i}=\left\{\left(1+L_{e} s / h_{0}\right) /\left(1+\beta^{4} \phi_{1}+\phi_{2}\right)\right\} \\
U_{e} / U_{r}=\left\{\left(1+L_{e} s / h_{o}\right)\left(1+\phi_{1}\right) /\left(1+\beta^{4} \phi_{1}+\phi_{2}\right)\right\}
\end{gathered}
$$

and

$\left.Q_{e} / Q_{i}=\beta^{2}\left(U_{e} / U_{1}\right)=\beta^{2}\left\{\left(1+L_{e} s / h_{0}\right) / 1+\beta^{4} \phi_{1}+\phi_{2}\right)\right\}^{1 / 2}$

$$
Q_{e} / Q_{r}=\beta^{2}\left(U_{e} / U_{r}\right)=
$$

$$
\beta^{2}\left\{\left(1+L_{e} s / h_{0}\right)\left(1+\phi_{1}\right) /\left(1+\beta^{4} \phi_{1}+\phi_{2}\right)\right\}^{1 / 2}
$$

Equations (6) through (11) are applied to examine the effects of variations of any of the drain pipe variables on $U$. and $Q_{n}$, as described in the following sections. 


\subsection{Effects of variations of the exit pipe diameter, $d$.}

For examining the effects of $d_{e}$ on $U_{e}$ and $Q_{e}$ the following simplification can be made without loss of generality: (1) $s$ is equal to zero; and (2) drain pipe is very smooth and has a short length so that the quantity $\left(f_{e} L_{e} / d_{e}\right)$ is negligible in comparison with the quantity $k_{c}$ in dissipation function $\phi_{2}$ that is $\phi_{2}=k_{c}$.

The value of $k_{c}$ is dependent upon the diameter ratio. For $\beta$ less than 1 (i.e. $d_{e}<d_{1}$ ) the value of $k_{c}$ has been experimentally determined by several researchers and may be found in the literature, typical values are shown in table 1 . For $\beta>1$ the relationship between $k_{c}$ and $\beta$ is given by the following expression:

$$
K_{\mathrm{c}}=\left(\beta^{2}-1\right)^{2}, \beta>1
$$

Variations of the quantities $U_{e} / U_{i}, U_{e} / U_{r}, Q_{e} / Q_{i}$ and $Q_{e} / Q_{r}$ due to variations in $d_{e} / d_{1}$, for 3 different representative values of $\phi_{1}$, are presented in graphical form in figures 4 and 5 .

TABLE 1

Values of loss coefficient, $K_{\text {, }}$ for sudden change in pipe diameter, taken from reference 7

\begin{tabular}{lllllllllll}
\hline$\beta^{2}$ & 0.1 & 0.2 & 0.3 & 0.4 & 0.5 & 0.6 & 0.7 & 0.8 & 0.9 & 1.0 \\
$K_{r}$ & 0.37 & 0.35 & 0.32 & 0.27 & 0.22 & 0.17 & 0.10 & 0.06 & 0.02 & 0.00 \\
\hline
\end{tabular}

In the case of velocity ratios, in figure 4 , it is seen that as the diameter ratio increases the velocity ratio decreases toward zero. As the drain pipe diameter increases for a fixed initial pipe diameter the velocity in the drain pipe will decrease or as the initial pipe diameter decreases for a fixed drain pipe diameter the velocity in the drain pipe will decrease. In figure 5 the efflux rate ratio $Q_{e} / Q_{r}$ goes to unity after obtaining a local maximum. The ratio $Q_{e} / Q_{i}$ reaches different asymptotic levels in the limit because the higher losses result in lower efflux rate as compared with the no loss condition required by $Q_{i}$. The critical value of diameter ratio $\beta_{c}$, at which $Q_{e} / Q_{r}$ is maximum is determined by setting

$$
\frac{d}{d \beta}\left(Q_{e} / Q_{r}\right)=0
$$

which yields

$$
\beta_{c}=\sqrt{2}
$$

The value of $Q_{e} / Q_{i}$ will also have the maximum value at $\beta_{c}$.

\subsection{Effects of drain pipe length}

To examine the effect of exit pipe length on the efflux the pipe slope, $s$, is assumed equal to zero and $d_{t}$ equal to $d_{e}$.

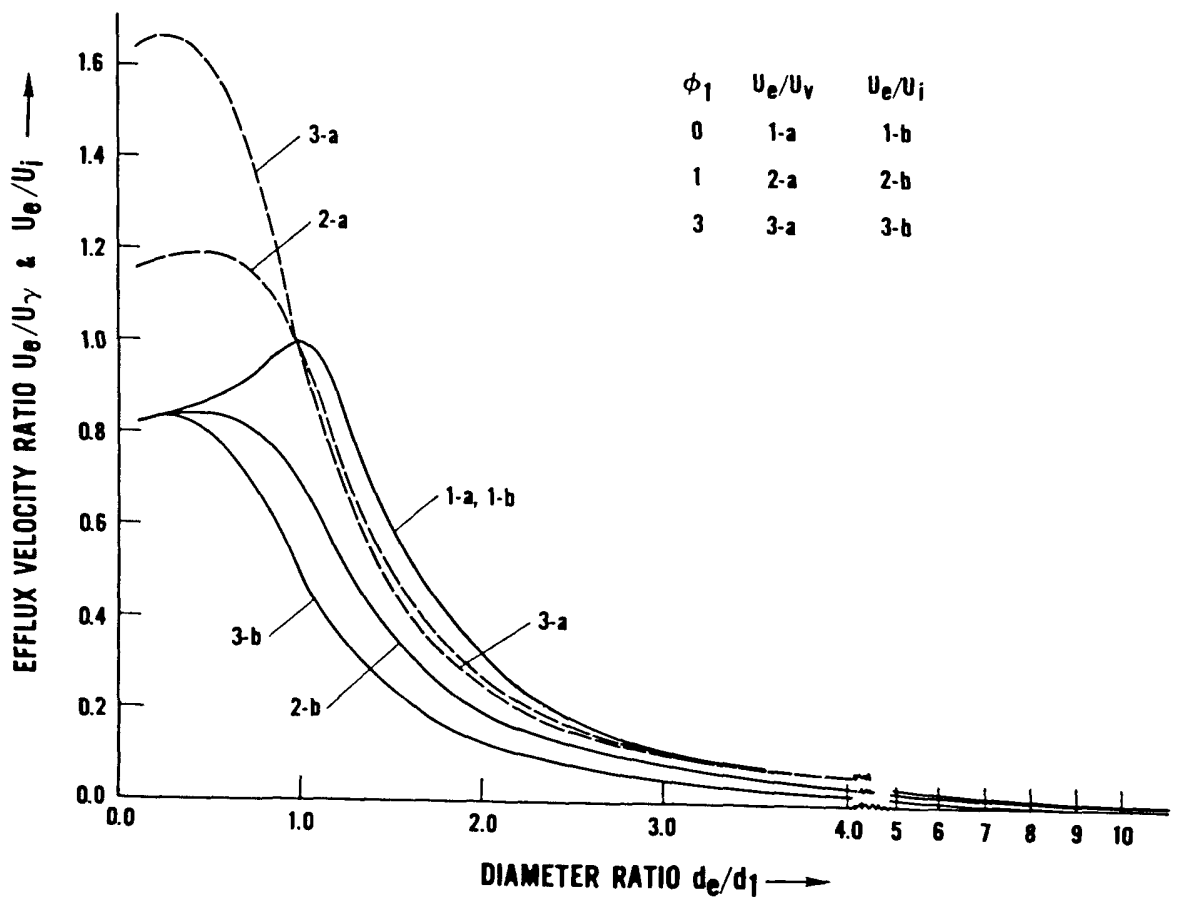

FIGURE 4. Water velocity ratio versus drain diameter ratio for efflux from a constant head container. 


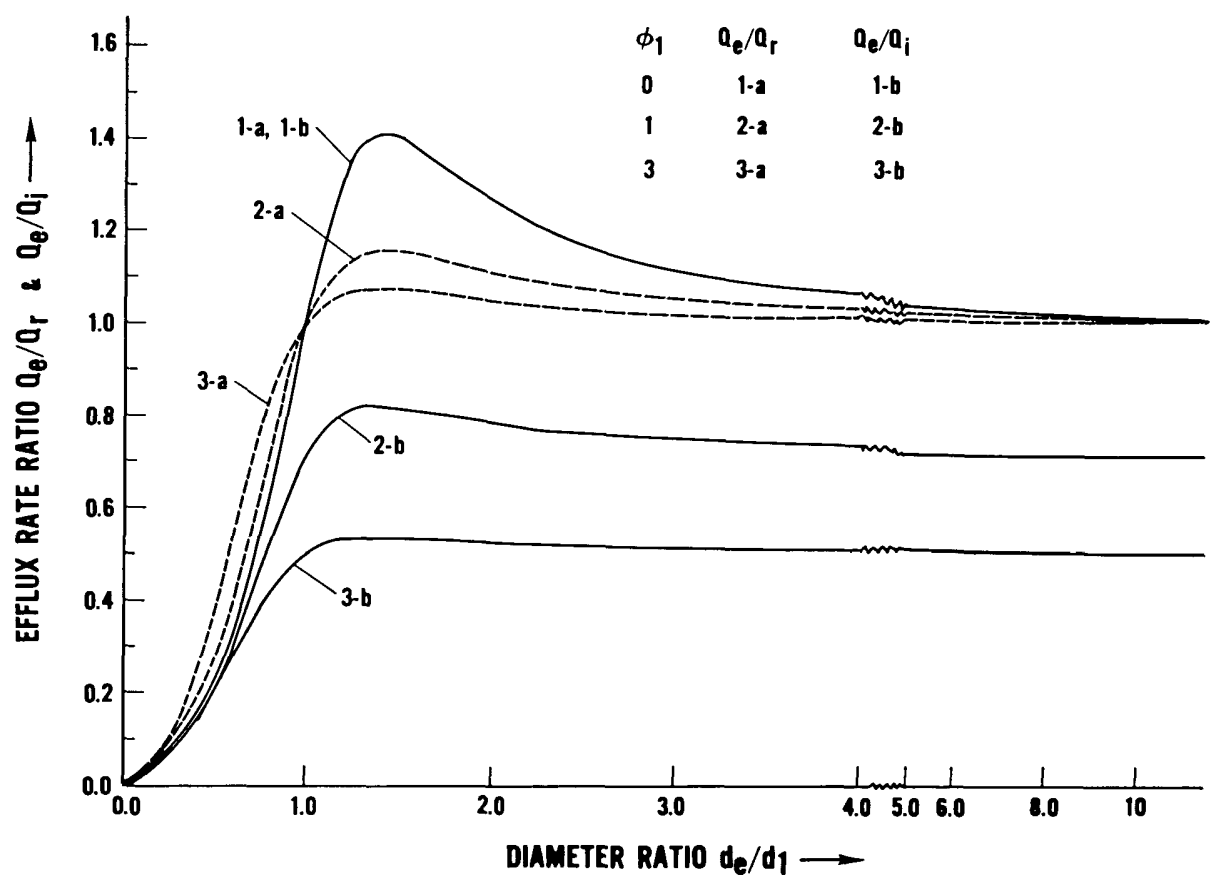

FigURE 5. Efflux rate ratio versus drain diameter ratio for efflux from a constant head container.

By substitution and rearranging terms, the efflux rate equation becomes:

$$
Q_{e} / Q_{r}=\left\{1 /\left[1+f_{e} L_{e} / d\left(1+\phi_{1}\right)\right]\right\}^{1 / 2}
$$

The variations of $Q_{e} / Q_{r}$ due to variation in drain pipe length are presented in figure 6 for these representative values of $\phi_{1}$, and for one value of $f_{e}(0.01)$.

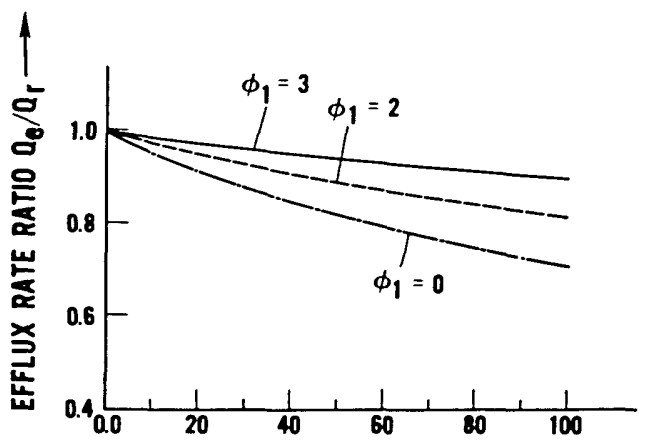

LENGTH TO DIAMETER RATIO $\left(\mathrm{Le}_{e} / \mathrm{d}\right) \longrightarrow$

Ficure 6. Efflux rate ration versus drain length to diameter ratio for efflux from a constant head container.

\subsection{Effects of drain pipe slope}

For this case with diameters $d_{1}$ equal to $d_{e}$, the equations may be simplified to illustrate the effects of changes in pitch. If the drain pipe is short and very smooth, so that quantity $f_{e} L_{e} / d$ is negligibly small in comparison to the quantity $\left(1+\phi_{1}\right)$, then the equations are further reduced:

$$
Q_{e} / Q_{r}=U_{e} / U_{r}=\left[\left(1+L_{e} s / h_{0}\right) /\left(1+\phi_{1}\right)\right]^{1 / 2}
$$

and

$$
Q_{e} / Q_{r}=U_{e} / U_{r}=\left[1+L_{e} s / h_{o}\right]^{1 / 2}
$$

The variations of $Q_{e} / Q_{r}$ due to variations in $s$, for three different values of $L_{e} / h_{o}$, are shown in figure 7 . The increase in efflux with increasing pitch is consistent with the effect of the larger gravity force component that accelerates the flow. For the longer pipe a greater influence is obtained.

\subsection{Effects of drain pipe friction factor}

For examining only the effects of pipe friction on the flow it is assumed that $s$ is equal to zero and $d_{1}$ is equal to $d_{e}$. The simplified equations become: 


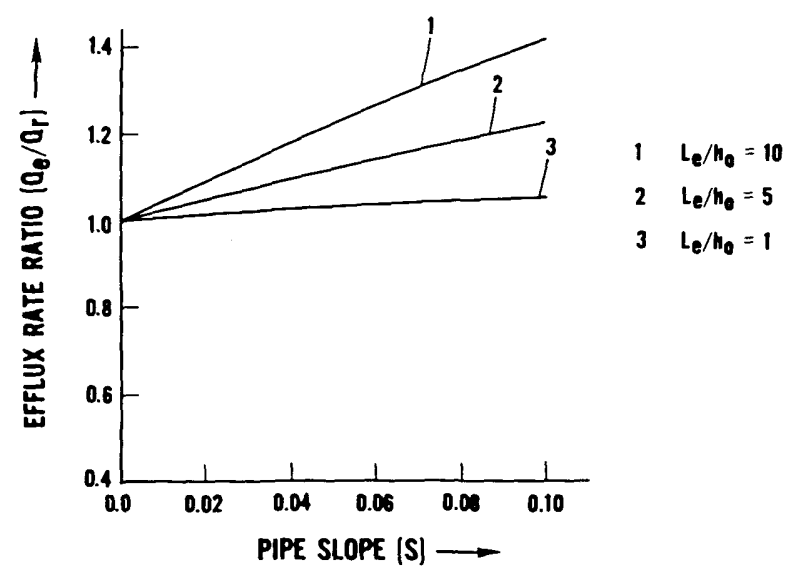

Ficure 7. Efflux rate ratio versus pipe slope for efflux from a constant head container.

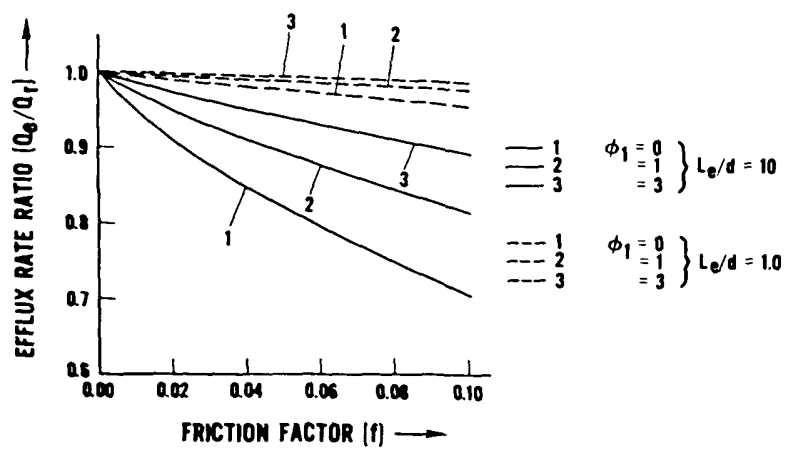

Ficure 8. Efflux rate ratio versus pipe friction factor for efflux from a constant head container.

$$
U_{e} / U_{i}=Q_{e} / Q_{r}=\left\{1+L_{e} s / h_{o}\right\}^{1 / 2}
$$

and

$$
U_{e} / U_{r}=Q_{e} / Q_{r}=\left[(1+\phi) /\left(1+\phi_{1}+f_{e} L_{e} / d_{e}\right)\right]^{1 / 2}
$$

The variations of $Q_{e} / Q_{r}$ due to variation in $f_{e}$, for three different values of $\phi_{1}$ and two values of $L_{e} / d_{e}$, are shown in figure 8.

It is noted that for a given value of the friction factor the ratio $Q . / Q$, increases with increasing $\phi_{1}$. The physical interpretation is that increased losses at the tank drain exit and the initial pipe section without the added length rapidly decrease $Q_{r}$. In terms of actual efflux rate, $Q_{e}$, the rate of flow is actually falling off with increased losses, but at a reduced rate as compared to $Q_{r}$.

\section{Effects of drain variables on the fall of water level and time needed to empty a container}

The depth of water and time required to empty the container of figure 3 is considered under quasi-steady flow conditions analagous to the region of slowly varying velocity shown in figure 2. When the free surface in the container is at height, $h$, above the center of the exit cross-section of the drain pipe, the instantaneous mean velocity, $U_{e}$, is given by the relationship:

$$
U_{e}=\left[2 g h /\left\{1+\beta^{4} \phi_{1}+\phi_{2}\right\}\right]^{1 / 2}
$$

The continuity eq( 3 ) may be written as:

$$
-d h=\left(a_{e} / A\right) U_{e} d t
$$

To determine the time required to empty the container, eq (20) should be solved rigorously by a succession of integrations, each over a small range of $h$ and $U$, with approximate mean values for the time interval of the $\phi_{1}$ and $\phi_{2}$ values being used. This differs from the approach [6] where along a streamline of the flow the head varies with time and a solution is obtained based upon the exit pipe velocity. However, for examining the effects of the parameters on the time required to empty the container, the values of $\phi_{1}$ and $\phi_{2}$ are assumed constant and the equation is integrated in closed form following [6] with the initial condition $t=0, h=h_{o}$, which yields

$$
t=2\left(A / a_{e}\right)\left[\left(1+\beta^{4} \phi_{1}+\phi_{2}\right) / 2 g\right]^{1 / 2}\left(\sqrt{h_{e}}-\sqrt{ } h\right)
$$

where $t$ is the time required for the liquid level in the container to fall from $h_{e}$ to $h$. The time required to empty the container, $t_{e}$, is obtained by setting $h=h_{1}$ (see fig. 3).

The non-dimensionalized value with respect to $t_{r}$ is given by:

$$
t_{e} / t_{r}=\left(1 / \beta^{2}\right)\left[\left(1+\beta^{4} \phi_{1}+\phi_{2}\right) /\left(1+\phi_{1}\right)\right]^{1 / 2}
$$

where $t_{r}=\left(A / a_{1}\right)(2 / g)^{1 / 2}\left(\sqrt{ } h_{e}-\sqrt{ } h_{1}\right)\left(1+\phi_{1}\right)^{1 / 2}$ is the time required to empty the container when the drainage system consists only of the initial pipe.

The equations indicate that the time required to empty the container will increase when $\phi_{2}$ is increased and all of the other variables are kept constant. To examine the effect of the variation of $d_{c}$ on the time required to empty the container, the quantity $f_{e} L d d_{e}$ may be assumed negligible in comparison with the quantity $\left(1+k_{c}\right)$.

Another form of the equation is:

$\left(h-h_{1}\right) /\left(h_{e}-h_{1}\right)=1+\eta^{2} t^{2} /\left(h_{e}-h_{1}\right)-2 \eta \mathrm{t} \sqrt{ } h_{e} /\left(h_{e}-h_{1}\right)$ 


$$
\left(\eta=\left(a_{e} / 2 A\right)\left[2 g /\left(1+\beta^{4} \phi_{1}+\phi_{2}\right)\right]^{1 / 2} .\right.
$$

Equations (22) and (23) are presented graphically for different representative conditions, in figures 9 and 10 , respectively. Higher efflux rates are anticipated (as has been shown) with lower dissipation values, and consequently the time for discharge is smaller. The maximum discharge value for $\beta=\sqrt{2}$ results in the minimum time value shown in figure 9 .

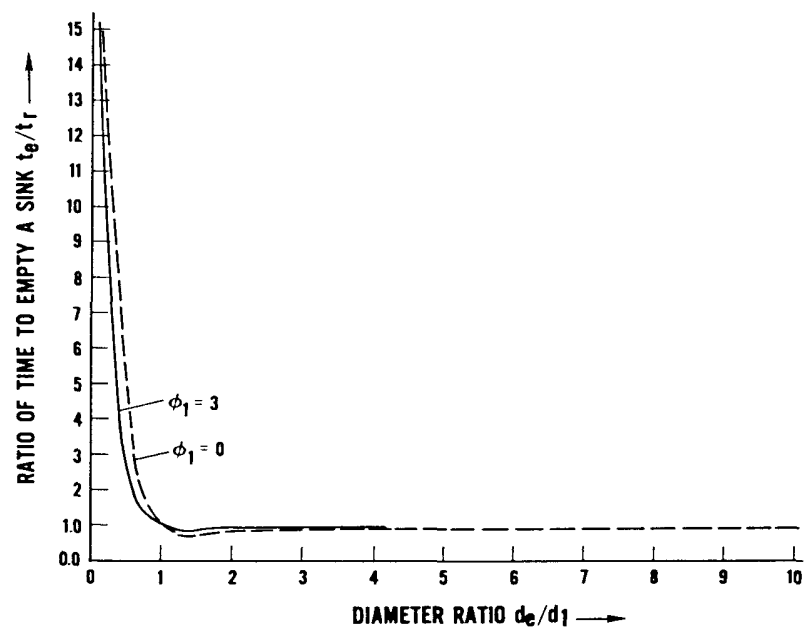

Figure 9. Time to empty sink versus drain diameter ratio.

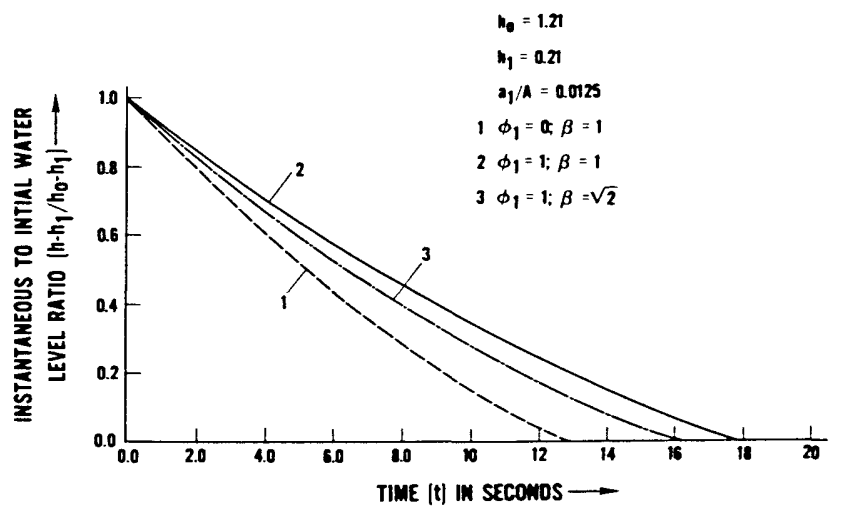

Figure 10. Water level ratio versus time for a falling head container.
Although the predictive models developed in this paper are for simple plumbing systems, the examples demonstrate the feasibility of developing mathematical models to predict the hydraulic characteristics and performance of complex plumbing systems. The theoretical discharge characteristics obtained by numerical methods of solution of the governing differential equation qualitatively resemble the experimental discharge characteristics for various fixtures reported by Wyly and Hintz [8] and Pink [9]. To obtain quantitative agreement between the predicted and experimental results it is necessary to determine the values of various loss coefficients from the experiments. The results presented showing the predicted variations of efflux rate with the diameter, length and slope of the drain pipe are similar to trends to the experimental results reported by Hanslin and Perrier [4].

\section{References}

[1] Hunter, Roy B. Methods of estimating loads in plumbing systems. NBS Building Materials and Structures Report. BMS 65, 1940.

[2] Eaton, H. N.; French, J. L. Fixture unit ratings as used in plumbing systems design. Housing and Home Finance Agency, Housing Reference Paper November 15, 1951.

[3] Konen, T. P.; Jackson, T.; Fowell, A. J. The Evaluation of several plumbing drainage waste and vent systems. Davidson Laboratory, Stevens Institute of Technology, Report SIT-DL-74-1776 (December 1974).

[4] Hanslin, R.; Perrier, F. The hydraulics of waste piping for sanitary appliances. Paper 12 presented at CIB W62 Seminar held in Stockholm, Sweden, 1974.

[5] Rouse, H. Elementary Mechanics of Fluids, New York: John Wiley and Sons; Fifteenth edition; 1964.

[6] Massey, B. S. Mechanics of Fluids, London: D. Van Nostrand; 1968.

[7] John, J. E. A.; Haberman, W. Introduction to Fluid Mechanics, Englewood Cliffs, NJ: Prentice Hall, Inc; 1971.

[8] Wyly, R. S.; Hintz, D. Report on the discharge characteristics of household plumbing fixtures. NBS Report 1131, 1951 .

[9] Pink, B. J. Laboratory investigation of the discharge characteristics of sanitary appliances. Paper CP37/73 presented at the CIB W62 Symposium Research Seminar No. 2, held at the National Swedish Building Research Institute, Stockholm, 1973. 